Mon. Not. R. Astron. Soc. 000, 10 (2002) Printed 26 November $2018 \quad$ (MN LATEX style file v2.2)

\title{
On the Origin of the Absorption Features in SS 433
}

\author{
A. D. Barnes ${ }^{1 \star}$, J. Casares ${ }^{2}$, P. A. Charles ${ }^{1,3}$, J. S. Clark ${ }^{4,5}$, R. Cornelisse ${ }^{1}$, \\ C. Knigge ${ }^{1}$ and D. Steeghs ${ }^{6}$ \\ ${ }^{1}$ School of Physics and Astronomy, University of Southampton, SO17 1BJ, UK \\ ${ }^{2}$ Instituto de Astrofisica de Canarias, 38200 La Laguna, Tenerife, Spain \\ ${ }^{3}$ South African Astronomical Observatory, PO Box 9, Observatory 7935, Cape, South Africa \\ ${ }^{4}$ Department of Physics and Astronomy, University College London, Gower Street, London, WC1E 6BT, UK \\ ${ }^{5}$ Department of Physics and Astronomy, The Open University, Walton Hall, Milton Keynes, MK7 6AA, UK \\ ${ }^{6}$ Harvard-Smithsonian Center for Astrophysics, MS-67, 60 Garden Street, Cambridge, MA 02138, USA
}

\begin{abstract}
We present high-resolution optical spectroscopy of the X-ray binary system SS 433, obtained over a wide range of orbital phases. The spectra display numerous weak absorption features, and include the clearest example seen to date of those features, resembling a mid-A type supergiant spectrum, that have previously been associated with the mass donor star. However, the new data preclude the hypothesis that these features originate solely within the photosphere of the putative mass donor, indicating that there may be more than one region within the system producing an A supergiantlike spectrum, probably an accretion disc wind. Indeed, whilst we cannot confirm the possibility that the companion star is visible at certain phase combinations, it is possible that all supergiant-like features observed thus far are produced solely in a wind. We conclude that great care must be taken when interpreting the behaviour of these weak features.
\end{abstract}

Key words: binaries: close - stars:binaries:individual:SS 433 - stars:winds,outflows binaries:spectroscopic

\section{INTRODUCTION}

The bizarre object SS 433 is a galactic X-ray binary at the centre of the supernova remnant W 50. It is observed as a weak $\left(\sim 10^{36} \mathrm{erg} \mathrm{s}^{-1}\right)$ X-ray source $(\mathrm{X} 1909+048)$, which combined with the high orbital inclination $\left(i \sim 78^{\circ}\right)$ and low $\mathrm{L}_{X} / \mathrm{L}_{\text {opt }}$ ratio $(V \sim 14.2)$ suggests that it may be an accretion disc corona source. In addition to its pair of relativistic jets $(\mathrm{v} \sim 0.26 \mathrm{c})$, recent radio observations have detected extended emission in a direction that is perpendicular to the main jet outflow (Blundell et al. 2001). This provides evidence for some form of circumbinary outflow such as a disc-like outflow of matter from the outer parts of the accretion disc (Zwitter et al. 1991). The overall system geometry suggested by these recent studies is illustrated in Fig. 11 a, a schematic that we will use extensively throughout this paper.

The system displays two key periodicities, a $\sim 162 \mathrm{~d}$ precession period of its jets and $\mathrm{a} \sim 13 \mathrm{~d}$ orbital period. The precession of the jets is revealed in the radial velocity curves of the strong, blue- and red-shifted 'moving' Balmer emission lines (Margon 1984). This 162d modulation is also present in the optical flux, a result of the changing orientation of the precessing accretion disc. The orbital period was first discovered through the radial velocity variations of the 'stationary' Balmer and HeI emission lines (Crampton et al. 1980), which reach maximum velocity at superior conjunction of the X-ray source. However, the He II $\lambda 4686$ has a different radial velocity curve, with a velocity maximum occurring when the compact object is receding (Crampton \& Hutchings 1981; Fabrika \& Bvchkova 1990). This may indicate an origin in or around the accretion disc/compact object.

Due to the strong and broad emission lines, the detection of any spectroscopic signatures of the donor star has proved rather more difficult, and the suggestions for its spectral type range from OB to WolfRayet (Crampton \& Hutchings 1981; van den Heuvel et al. 1979). Since the interpretation of the radial velocity curves requires assumptions about the mass donor, estimates for the mass of the compact object have ranged as widely as $0.8 M_{\odot}$ (d'Odorico et al. 1991) and $62 M_{\odot}$. (Antokhina \& Cherepashchuk 1985). Gies et al. (2002b) and Hillwig et al. (2004) suggested that the optimal time to detect a signature of the donor star is during the X-ray eclipse $\left(\phi_{\text {orb }} \sim 0\right)$, at a precessional phase when the disc 
is most open to the observer $\left(\Psi_{\text {prec }} \sim 0\right.$, Fig. 1b). During this configuration of phases, light from the donor should be least 'obscured' by the extended circumbinary disc or disc wind. Based on observations taken during this combination of phases, Hillwig et al. (2004) discovered features corresponding with a mid-A supergiant (SG) and suggested that these could originate upon the donor star. This constrained the binary system to be a low mass black hole $(2.9$ $\left.\pm 0.7 M_{\odot}\right)$ with a $10.9 \pm 3.1 M_{\odot}$ companion, consistent with the predictions of King et al. (2000).

However, these calculations were based upon limited orbital phase coverage. In this paper we will test the possibility of observing the A SG features over a much broader range of phases in order to confirm their origin and provide a more secure estimate of the system parameters. In Section 2 we give an overview of the observations. In Section 3 we will begin with a general description of all our spectra, then discuss the three best spectra in more detail (Sections 3.1-3.3), and finish by presenting the radial velocities in Section 3.4. We will begin Section 4 with a discussion of the consequences of the model proposed by Gies et al. (2002b) and then discuss our findings.

\section{OBSERVATIONS}

SS 433 was observed using three different telescopes and instruments in the blue spectral range $(\lambda \lambda 4000-5000)$ between 2000 and 2004. Four high-resolution spectra of SS 433 were obtained with the TWIN spectrograph mounted on the $3.5 \mathrm{~m}$ telescope at Calar Alto observatory. A 1200 lines $\mathrm{mm}^{-1}$ grating together with a $1.5^{\prime \prime}$ long slit covered $\lambda \lambda 3700$ 4800 at $0.51 \AA$ pix $^{-1}$, yielding a spectral resolution of $67 \mathrm{~km}$ $\mathrm{s}^{-1}$ (FWHM) at $4550 \AA$. Two intermediate resolution spectra $\left(0.63 \AA \mathrm{pix}^{-1}\right)$ were obtained using the Intermediate Dispersion Spectrograph (IDS) attached to the $2.5 \mathrm{~m}$ Isaac Newton Telescope (INT) at the Observatorio del Roque de Los Muchachos on the nights of 15-16 August 2002. We used the R900V grating in combination with the $235 \mathrm{~mm}$ camera and a $1.2^{\prime \prime}$ slit to provide a spectral resolution of $83 \mathrm{~km} \mathrm{~s}^{-1}$ (FWHM) at $4550 \AA$.

Nine high resolution spectra of SS 433 in addition to an A SG comparison star were also obtained using the blue arm of the ISIS spectrograph with the $4.2 \mathrm{~m}$ William Herschel Telescope (WHT) at the Observatorio del Roque de Los Muchachos in June/July 2004. The R1200B grating was used with a central wavelength setting of $4550 \AA$ and a $0.93^{\prime \prime}$ slit, giving a spectral resolution of $29 \mathrm{~km} \mathrm{~s}^{-1}$ (FWHM) $4550 \AA$. A complete log of the observations is presented in Table 1 Orbital phases have been calculated using the lightcurve ephemeris of Goranskii et al. (1998), whilst disk precession phases are based on the ephemeris of Gies et al. (2002a).

The images were bias corrected and flat-fielded, and the spectra subsequently extracted using conventional optimal extraction techniques in order to optimize the signal-to-noise ratio of the output (Horne 1986). Frequent observations of comparison arc lamp images were performed in the course of each run and the pixel-to-wavelength scale was derived through polynomial fits to a large number of identified reference lines. The final rms scatter of the fit was always $<1 / 30$ of the spectral dispersion. Finally, the spectra have been rec- tified using a high-pass filter to remove any low-order variations (0.001-0.08 cycles $/ \AA)$ caused by the broad jet lines, and then the data were combined to produce an average spectrum for each night.

\section{ANALYSIS}

We analysed a portion of the continuum that is free of stationary emission lines and rich in absorption features. The best available region encompasses the range between $\sim 4500$ - $4630 \AA$, which is bracketed by emission from He I $\lambda 4471$ and the N III/C III complex 4640-4650 А.

Spectra from twelve of the fifteen separate nights, covering a broad range of orbital phases, display photospheric features that appear to be similar to that of an A SG (Fig. 2). Whilst the strongest Fe II absorption lines $(\lambda 4549 \& \lambda 4584$ $\AA)$ are present in each of these spectra, the morphology of these features, and indeed the presence of other weaker features, is highly variable, a fact which seems difficult to reconcile with the donor star being the only system component producing these lines.

We have extracted velocity measurements from each of the spectra which display clear A SG-like photospheric features by cross-correlating with an optimally broadened comparison star (HD 9233). The systemic velocity of the comparison star was first removed $\left(\gamma=-34 \pm 2 \mathrm{~km} \mathrm{~s}^{-1}\right.$, Hillwig et al. 2004) and then the data were re-binned into velocity space and cross-correlated with the optimally broadened spectrum of HD 9233 across the range $\sim 4500-4630 \AA$. The comparison star was smoothed to the resolution of the spectra for the Calar Alto and INT runs using a Gaussian smoothing algorithm. We present these results in Section 3.4, after a more detailed description and comparison of three of these spectra which display the clearest absorption lines.

For each nightly average spectrum, we broadened our A SG template from 39 to $99 \mathrm{~km} \mathrm{~s}^{-1}$ in steps of $2 \mathrm{~km}$ $\mathrm{s}^{-1}$, taking into account that the template star HD 9233 already possesses an intrinsic rotational broadening of 39 $\mathrm{km} \mathrm{s}^{-1}$ (Hillwig et al. 2004). A spherical rotational profile Grav 1992) was applied, with a linear limb-darkening law of coefficient $\epsilon=0.62$, interpolated for $\lambda=4500 \AA$ and $T_{\text {eff }} \simeq 8350 \mathrm{~K}$ (Kudritzki et al. 2003; Al-Naimv 1978). The broadened versions of the template star were multiplied by fractions $f<1$, to account for the fractional contribution to the total light, and subsequently subtracted from the nightly average spectra for SS 433. Minimising $\chi^{2}$ yields an optimal value for $f$ and $v \sin i$ in each case (see Table 1 ).

\subsection{A supergiant spectrum in SS 433?}

As can be seen in Fig. 3] ( $\left.\Psi_{\text {prec }} \sim 0.66, \phi_{\text {orb }} \sim 0.78\right)$, we have a remarkable match between the SS 433 spectrum and the A4 Iab comparison star. All of the key absorption lines present in this region of the comparison star also manifest themselves in our spectrum of SS 433, though broader and somewhat shallower, as might be expected due to the continuum emission of the accretion disc. A $\chi^{2}$ test on the residuals yields $v \sin i=84 \pm 5 \mathrm{~km} \mathrm{~s}^{-1}$, whilst the depth of the lines are equivalent to an optimal scaling factor of $0.77 \pm 0.04$. Cross-correlating with the comparison star HD 9233 results in a velocity shift measurement of $6 \pm 2 \mathrm{~km} \mathrm{~s}^{-1}$. 


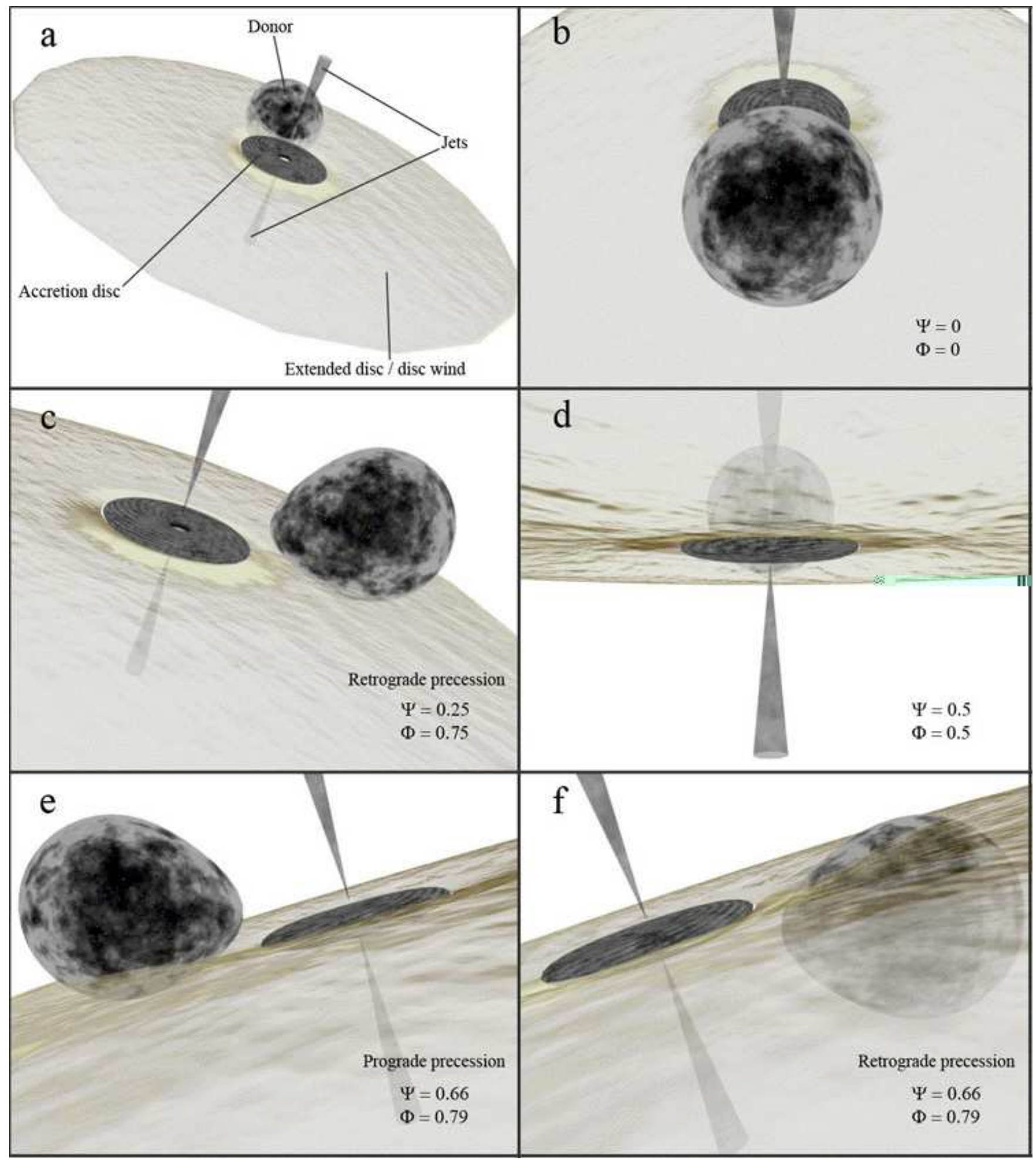

Figure 1. Cartoon picture of the SS 433 system with an equatorially expanding extended disc, from the point of view of an observer on earth. In all panels the sense of precession is represented as being anti-clockwise, with orbital motion clockwise in the retrograde case and anti-clockwise in the prograde case. a) Overview. Note the extended disc is arbitrarily cut-off in the radial direction, and its opening angle is rather narrow, neither of which are confirmed physical parameters. b) Preferred phase combination of Hillwig et al. (2004). Donor could still be visible above the extended disc from $\phi_{\text {orb }} \sim 0.75-0.25$. c) Later $\Psi_{\text {prec }}$, but the donor could still be visible above the extended disc at this combination of phases. $d$ ) Donor star becomes fully masked behind the extended disc, but will become partially visible below the disc from $\phi_{\text {orb }} \sim 0.75-0.25$. e) \& f) The system alignment on 2004 June 29 . Here the donor is mostly visible assuming prograde precession, and masked in the case of retrograde (the preferred model). 
Table 1. Observing log of our SS 433 spectroscopy, and derived parameters. For each observation we give the orbital phase, $\phi_{\text {orb }}$, and precessional phase, $\Psi_{\text {prec }}$, at the time of the observation. We also give the radial velocities obtained via cross-correlation fitting, the estimated scaling factor of the absorption lines compared to a mid-A SG comparison star, $f$, and the optimal broadening applied to the comparison star, $v \sin i$ (see Sect. 3). The spectra from 2000-07-23, 2004-07-02 and 2004-07-04 do not display clear A SG-like features.

\begin{tabular}{|c|c|c|c|c|c|c|c|c|c|}
\hline Date & $\begin{array}{c}\text { HJD } \\
-2450000\end{array}$ & Obs. & $\begin{array}{l}\text { Exp. Time } \\
\text { (seconds) }\end{array}$ & $\begin{array}{l}\text { Reciprocal Dispersion } \\
\left(\AA \mathrm{pix}^{-1}\right)\end{array}$ & $\phi_{o r b}$ & $\Psi_{\text {prec }}$ & $\begin{array}{l}\text { Radial Velocity } \\
\qquad\left(\mathrm{km} \mathrm{s}^{-1}\right)\end{array}$ & $f$ & $\begin{array}{c}v \sin i \\
\left(\mathrm{~km} \mathrm{~s}^{-1}\right)\end{array}$ \\
\hline 2000-07-20 & 1746.38 & C.A. & 1800 & 0.51 & 0.687 & 0.777 & $-54 \pm 15$ & $0.80 \pm 0.09$ & $97 \pm 30$ \\
\hline 2000-07-21 & 1747.43 & C.A. & 1800 & 0.51 & 0.768 & 0.784 & $-91 \pm 16$ & $1.03 \pm 0.10$ & $110 \pm 20$ \\
\hline $2000-07-22$ & 1748.36 & C.A. & 1800 & 0.51 & 0.839 & 0.789 & $-20 \pm 22$ & $1.04 \pm 0.05$ & $108 \pm 15$ \\
\hline 2000-07-23 & 1749.42 & C.A. & 1800 & 0.51 & 0.920 & 0.796 & - & - & - \\
\hline 2002-08-15 & 2501.64 & INT & 1800 & 0.63 & 0.420 & 0.435 & $-120 \pm 5$ & $0.51 \pm 0.05$ & $80 \pm 8$ \\
\hline 2002-08-16 & 2502.55 & INT & 1800 & 0.63 & 0.490 & 0.441 & $-115 \pm 12$ & $0.29 \pm 0.07$ & $77 \pm 10$ \\
\hline 2004-06-29 & 3186.67 & WHT & $3 \times 1800$ & 0.22 & 0.784 & 0.660 & $6 \pm 2$ & $0.77 \pm 0.04$ & $84 \pm 5$ \\
\hline 2004-06-30 & 3187.63 & WHT & $3 \times 1800$ & 0.22 & 0.857 & 0.666 & $-5 \pm 8$ & $0.46 \pm 0.08$ & $98 \pm 17$ \\
\hline 2004-07-01 & 3188.67 & WHT & $3 \times 1800$ & 0.22 & 0.937 & 0.672 & $4 \pm 13$ & $0.65 \pm 0.05$ & $39 \pm 4$ \\
\hline 2004-07-02 & 3189.68 & WHT & $3 \times 1600$ & 0.22 & 0.014 & 0.678 & - & - & - \\
\hline 2004-07-03 & 3190.51 & WHT & $3 \times 1800$ & 0.22 & 0.077 & 0.683 & $-12 \pm 13$ & $0.30 \pm 0.06$ & $53 \pm 8$ \\
\hline 2004-07-04 & 3191.63 & WHT & $3 \times 1800$ & 0.22 & 0.163 & 0.690 & - & - & - \\
\hline 2004-07-20 & 3207.43 & WHT & $6 \times 600$ & 0.22 & 0.371 & 0.788 & $-78 \pm 8$ & $0.10 \pm 0.06$ & $73 \pm 20$ \\
\hline 2004-07-30 & 3217.43 & WHT & $3 \times 1800$ & 0.22 & 0.135 & 0.849 & $-1 \pm 5$ & $0.59 \pm 0.04$ & $49 \pm 4$ \\
\hline 2004-07-31 & 3218.50 & WHT & $3 \times 1800$ & 0.22 & 0.217 & 0.856 & $-35 \pm 4$ & $1.05 \pm 0.08$ & $117 \pm 10^{*}$ \\
\hline
\end{tabular}

* Measurement of the combined features in the double-lined spectrum (see Sect. 3.3)

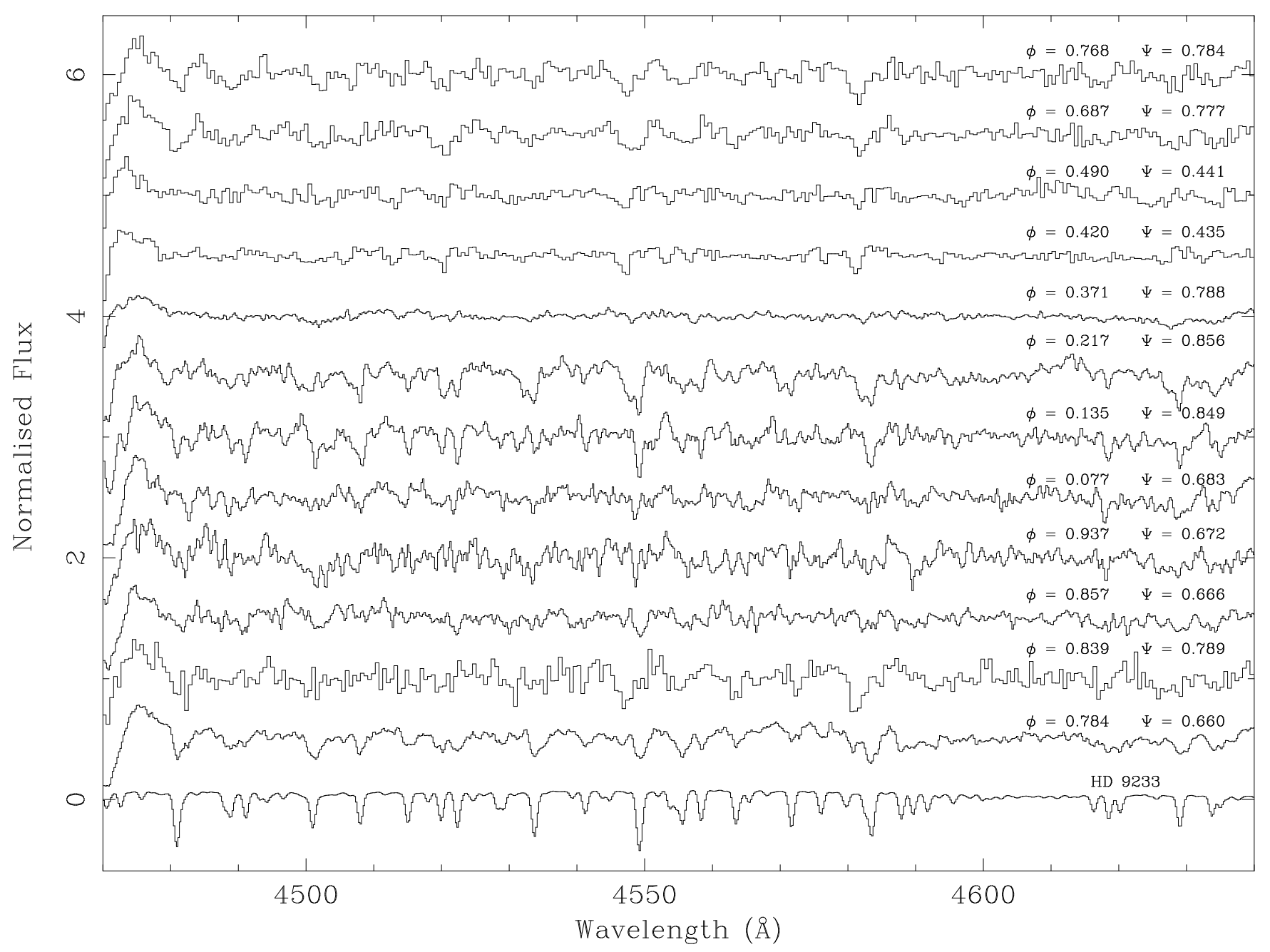

Figure 2. All of the nightly averaged spectra which display key A supergiant-like features arranged in orbital phase order beginning with the spectrum discussed in Section 3.1 (second from bottom). The template star HD 9233 is shown at the bottom. Line identifications have been omitted here for clarity, see Figs. 31 4 for this information. 


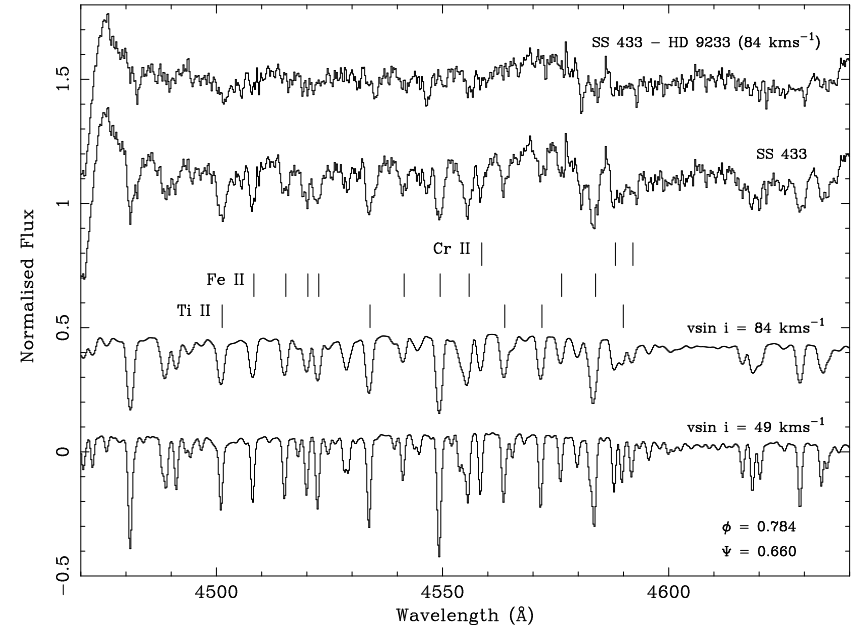

Figure 3. Average spectrum of SS 433 from 2004 June 29 $\left(\Psi_{\text {prec }} \sim 0.66, \phi_{\text {orb }} \sim 0.78\right)$, together with the template star HD 9233 broadened by different amounts (below), and the residual of SS 433 - optimally broadened template (top).

Subtracting the optimally broadened template star from the average spectrum of SS 433 provides a remarkably clean residual spectrum (Fig. 3. top), with the only unusual features in the region of interest being the He I $\lambda 4471 \mathrm{P}$ Cyg profile, and a blue-shifted jet line bump (He I $\lambda 4713$ ) which has not been fully eliminated during the high-pass filtering process.

\subsection{Narrow Absorption Lines}

The spectrum displayed in Fig. $4\left(\Psi_{\text {prec }} \sim 0.85, \phi_{\text {orb }} \sim\right.$ 0.14 ) is also very rich in the A SG-like features identified by Hillwig et al. (2004), including many FeII, Cr II and Ti II lines. However, it is immediately apparent even by eye that these absorption lines possess a much narrower and sharper profile $\left(49 \pm 4 \mathrm{~km} \mathrm{~s}^{-1}\right.$ compared to $\left.84 \mathrm{~km} \mathrm{~s}^{-1}\right)$ than those from the observation in Sect. 3.1.

The fractional contribution of $0.59 \pm 0.04$ is somewhat lower too, indicating that a large proportion of the light in this spectral region, at this particular time, may be produced elsewhere in the system, e.g. the accretion disc.

The family of absorption lines are slightly blue-shifted compared to the observation discussed in Sect. 3.1, with a velocity of $-1 \pm 5 \mathrm{~km} \mathrm{~s}^{-1}$. If these lines are coming from the donor star then this is not the expected behaviour, which should have been close to its maximum blue-shift in the earlier observation, and approaching maximum red-shift at this orbital phase.

\subsection{Double Absorption Lines}

Even more anomalously, the spectrum from the following night $\left(\Psi_{\text {prec }} \sim 0.86, \phi_{\text {orb }} \sim 0.22\right.$, Fig. 5) appears to possess absorption lines which are doubled (Fe II and Ti II), with a narrower red component blended with a broader, shallower blue. This feature is evident in each of the individual spectra from the night, and is not therefore the signature of some systematic error introduced when producing the average spectrum for the night.

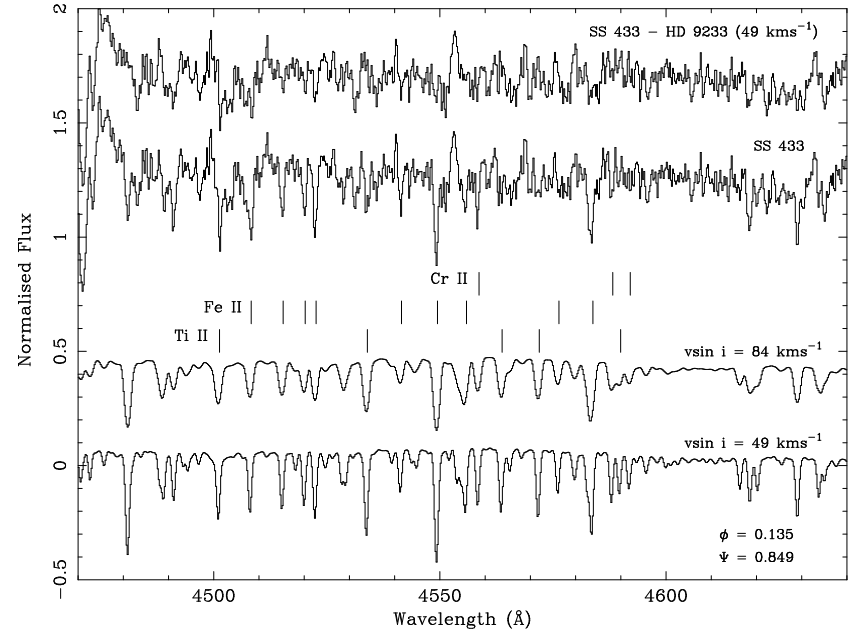

Figure 4. Average spectrum of SS 433 from 2004 July 30 $\left(\Psi_{\text {prec }} \sim 0.85, \phi_{\text {orb }} \sim 0.14\right)$. The template star HD 9233 is shown below for comparison, broadened by different amounts, and the residual of SS 433 - optimally broadened template (top). The emission feature at $\sim 4475 \AA$ is a component of the He I $\lambda 4471 \mathrm{P}$ Cyg profile (see text).

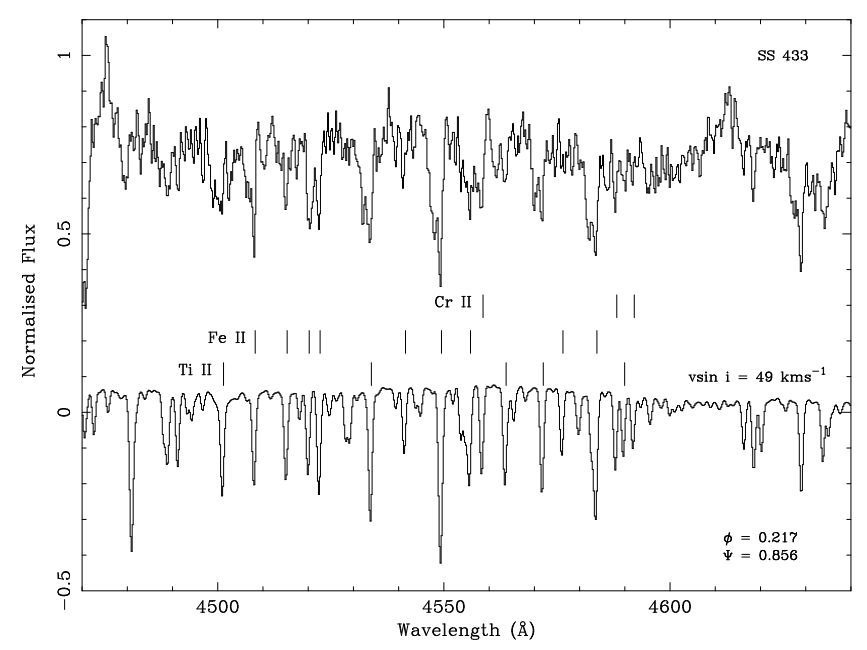

Figure 5. Average spectrum of SS 433 from 2004 July 31 $\left(\Psi_{\text {prec }} \sim 0.86, \phi_{\text {orb }} \sim 0.22\right)$. Note in particular the doubled absorption lines of Fe II and Ti II. Again, the template star HD 9233 is shown for comparison below, broadened to the optimal value from the previous night's observations.

Cross-correlating the data from this night against the A SG comparison star gives a velocity of $-35 \pm 4 \mathrm{~km} \mathrm{~s}^{-1}$. This is again much bluer than expected, with the observation coinciding with the expected maximal red-shift of the donor star. However, the cross-correlation technique in this case fails to fit either of the apparent absorption components in this spectrum, instead picking out an 'in-between' velocity. Gaussians fitted individually to the red and blue features indicate velocities of $-10 \pm 10$ and $-100 \pm 15 \mathrm{~km} \mathrm{~s}^{-1}$. Neither component therefore possesses a velocity commensurate with the predicted behaviour of the donor star. 


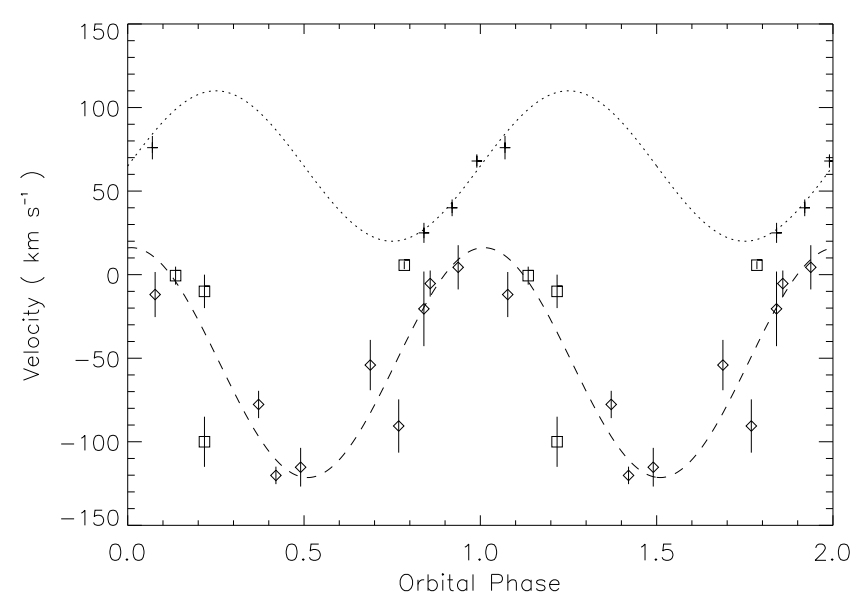

Figure 6. The radial velocities obtained via cross-correlation fitting in the $\lambda 4500-4600 \AA$ region. The results obtained by Hillwig et al. (2004) are shown marked by pluses, together with their best-fitting sine curve (dotted line). The remaining points are the results of our own campaign, with those marked by squares representing the spectra selected for discussion in Section 3. The dashed line represents the best-fitting sine curve to these data. Note that two orbits have been plotted for clarity.

\subsection{Absorption Line Velocities}

Our data cover a broad range of orbital phases, and are displayed in Fig. 6] as diamonds. The three spectra discussed in Sections 3.1-3.3 are marked with squares. Note that all the velocities used in this analysis were the result of crosscorrelation with a comparison star, with the exception of the individual line fits discussed in Section 3.3. The results reported by Hillwig et al. (2004) are indicated by pluses, with the dotted line representing their best-fitting sine curve to these data. The best-fit sine curve to our data is marked by a dashed line, with semi-amplitude $\mathrm{K}=69 \pm 4 \mathrm{~km} \mathrm{~s}^{-1}$, systemic velocity $\gamma=-53 \pm 3 \mathrm{~km} \mathrm{~s}^{-1}$ and with velocity maxima occuring at orbital conjunction, $0.26 \pm 0.01$ out of phase with the motion of the accretion disc (Fabrika \& Bvchkova 1990).

\section{DISCUSSION}

\subsection{A model for SS 433}

Gies et al. (2002b) and Hillwig et al. (2004) state that the mass donor is ideally observed around the primary eclipse and when the accretion disc has precessed to its most face-on position $\left(\Psi_{\text {prec }} \sim \phi_{\text {orb }} \sim 0\right)$. The donor star would then have the highest chance of being positioned above and between any extended disc and an observer (Fig. 1b). Extending this idea, it should also be possible to detect the donor star at certain other combinations of phases. In Fig. 1n we show an example, with the companion being positioned clearly above the extended disc, whilst in Fig. 11 $l$ we have the inverse situation, with the companion star being totally obscured behind the extended disc. However, at this $\Psi_{\text {prec }}$ the companion could become partially visible below the extended disc from $\phi_{\text {orb }} \sim 0.75-0.25$. The extent to which we may be able to observe the companion will be determined by the opening angle and radial size of the extended disc, parameters which are currently not well constrained, in addition to the degree of irradiation of the inner face of the donor star from the accretion disc (Cherepashchuk et al. 2005).

If this scenario is correct, it can provide direct observational information about the precession evident in the system. Confirming whether it be prograde or the preferred retrograde model (Newsom \& Collins 1982) with respect to the donor star could perhaps cast further light upon the physics driving the precession (Katz 1981; Leibowitz 1984). From Figs. 1 $e$ \& $f$, representing the system alignment of the spectrum discussed in Section 3.1, it becomes clear that the companion star could only be visible above the disc at this combination of phases if the precession were prograde in nature, with the extended disc masking the donor in the case of retrograde precession.

The presence of the same A SG-like features reported by Hillwig et al. (2004) in many of our spectra, at a range of different orbital and precessional phases, would at first glance seem to indicate that our data support this scheme. In particular, the observation discussed in Section 3.1 (where the accretion disc is close to edge-on, $\left.\Psi_{\text {prec }} \sim 0.66, \phi_{\text {orb }} \sim 0.78\right)$ provides an unsurpassed match between an A4 Iab comparison star and SS 433 (Fig. 3). If these features really do originate upon the donor star, then the depth and strength of the lines are equivalent to a scaling factor of $0.77 \pm 0.04$. This would indicate a surprisingly high contribution to the total light at this combination of phases, compared to observations with the disc face-on, where Hillwig et al. (2004) find a fractional contribution of $0.36 \pm 0.07$.

We can compare our estimated scaling factor with that expected from observed light curves of SS 433 in a similar manner to Hillwig et al. (2004). The key assumption required is that the donor star is totally eclipsed by the disc at $\Psi_{\text {prec }}=0$ and $\phi_{\text {orb }}=0.5$, and at all other times both the donor and the accretion disc are contributing to the total light. The V-band light-curves of Zwitter et al. (1991) imply a potential fractional contribution for the donor star of roughly $0.70 \pm 0.15$ at $\Psi_{\text {prec }} \sim 0.66, \phi_{\text {orb }} \sim 0.78$. Whilst this prediction appears to be consistent with our observational data, we must note that there is a large degree of scatter evident in the observed precessional light curves, introducing a degree of systematic error into these calculations. Indeed, if we use the same method to predict the donor contribution at phase combination $\Psi_{\text {prec }} \sim \phi_{\text {orb }} \sim 0$ from the Zwitter et al. (1991) light curves, the resulting estimate is too high by a factor of three.

If we presume the absorption features noted in Section 3.1 to be a signature of the donor star, then retrograde precession may be immediately ruled out. However, the same features also occur in the spectra discussed in Sections 3.23.3. These observations were performed at a phase combination where the donor should be obscured assuming prograde precession, and visible in the retrograde case. In addition to this apparent conflict, we must also account for the unusual velocity behaviour of these weak absorption features.

\subsection{A disc wind origin?}

Cross-correlating our spectra of SS 433 with an A supergiant spectrum produces a velocity curve (Fig. 6] $\gamma=-53 \pm 3 \mathrm{~km}$ $\mathrm{s}^{-1}$ ) which is far removed in terms of systemic velocity from the value of $65 \pm 3 \mathrm{~km} \mathrm{~s}^{-1}$ reported by Hillwig et al. (2004), 
though it is close to the result of Gies et al. (2002b), where $\gamma=-44 \pm 9 \mathrm{~km} \mathrm{~s}^{-1}$. Additionally, it is almost exactly a quarter out of phase with the Fabrika \& Bvchkova (1990) He II curve (where $\gamma=-22 \pm 14 \mathrm{~km} \mathrm{~s}^{-1}$ ) which traces the motion of the compact object.

The occurence of a velocity maximum at orbital conjunction essentially precludes the possibility that these features were produced in the photosphere of the companion. Whilst it is extremely difficult to pin down a particular region within the system which could produce this velocity signature, it is interesting to note that this behaviour has been reported before. Gies et al. (2002a) find the 'stationary' $\mathrm{He} \mathrm{I}$ and $\mathrm{H} \alpha$ emission lines display a remarkably similar semi-amplitude and phasing to the A SG-like features investigated here. This is attributed to a clumpy wind outflow from the accretion disc rather than the disc itself or to a gas stream (Goranskii et al. 1997; Gies et al. 2002a). Although there is a large disparity in line widths between the stationary emission lines and our narrow absorption lines, if what we observe in SS 433 is a structured outflow then we would expect to see narrow absorption components from localised density enhancements in the wind. This would appear to indicate that for at least some combinations of orbital and precessional phases the A SG 'photospheric' features arise not from the donor but instead from a localised area that can be associated with the region where the $\mathrm{HeI}$ and $\mathrm{H} \alpha$ stationary emission lines are produced.

We also have some longer wavelength data collected during the 2004 observing campaign in La Palma with ISIS ( $\sim 8400-8900 \AA)$, to be published in a later paper. Here we identify heavily blended Ca II and weak N I absorption lines, which again correspond to the absorption features expected for an early-mid A SG. Interestingly, there appears to be a strong positive correlation between the strength of the $\mathrm{P}$ Cygni profiles seen in the Paschen lines and the strength of the CaII and N I lines, implying another link between traditional wind features and those typical of the proposed companion star.

The strongest evidence to date for an A SG donor is the data presented by Hillwig et al. (2004). They base their identification on three criteria which suggest an association with the donor, namely the radial velocity curve characteristics, the absorption line depths and the absorption line width. However, their data do not cover the key phases when there should be a turnover in velocity space $\left(\phi_{\text {orb }} \sim 0.25\right.$, $0.75)$, which would unambiguously associate the lines with the donor. Interestingly, a number of our own spectra which can be clearly associated with an outflow also meet at least two of the above criteria (see Table1), with only the velocity curve excluding an association with the donor.

The large offset between the systemic velocity detected by Hillwig et al. (2004) and in our data could be a potential problem for a purely circumbinary outflow origin. However, this velocity offset could be explained by a considerable cycle to cycle variation in the semi-amplitude of the 'stationary' emission lines originating in the accretion disc wind (Gies et al. 2002a). Another problem could be the doubled absorption lines shown in Fig. [5 This could perhaps be indicative of two entirely separate regions with identical spectral signatures, though neither component corresponds to a possible donor star origin. It seems more likely that two different components of a clumpy wind outflow could concurrently pass through the line-of-sight, as proposed by Gies et al. (2002a) and Lépine et al. (2000).

\section{CONCLUSIONS}

Our series of high-resolution optical spectra of SS 433 clearly display numerous typical A SG photospheric features, as reported by several authors and attributed to a putative mass donor. However, the velocities of these features do not correspond to the expected motion of the mass donor, but rather to the $\mathrm{H} \alpha$ and He I 'stationary' emission lines noted by Gies et al. (2002a) and assumed to originate in a clumpy, accretion-driven outflow.

Although the observations of Hillwig et al. (2004) are suggestive of an A SG-like donor, we cannot exclude the possibility that these features originate from the same highly variable accretion-driven wind. If these lines are consistent over a number of observations at $\Psi_{\text {prec }} \sim \phi_{\text {orb }} \sim 0$ then it would seem likely that they are a signature of the donor. In this case the large off-set in systemic velocity between the Hillwig et al. (2004) data and ours could be due to the velocity of the outflow towards our line of sight. However, if the lines originate exclusively in some form of outflow, then we would expect them to be rather variable in terms of width, depth, shape and velocity. In any case, considerable care must be taken to correctly interpret these features and thus determine the nature of the stellar components within the SS 433 system.

\section{ACKNOWLEDGMENTS}

We gratefully acknowledge A.B. Hill and D. Moss for artistic direction, and Ph. Podsiadlowski and the referee D.R. Gies for helpful comments. ADB is supported by a PPARC Studentship. JSC is supported by an RCUK Fellowship. DS acknowledges a Smithsonian Astrophysical Observatory Clay Fellowship. JC acknowledges support from the Spanish MCYT grant AYA2002-0036. Based on observations collected at the Centro Astronomico Hispano Aleman (CAHA) at Calar Alto, operated jointly by the Max-Planck Institut fur Astronomie and the Instituto de Astrofisica de Andalucia (CSIC). The INT and WHT are operated on the island of La Palma by the Isaac Newton Group in the Spanish Observatorio del Roque de los Muchachos of the Instituto de Astrofisica de Canarias. We gratefully acknowledge the use of the MOLLY package developed by T.R. Marsh.

\section{REFERENCES}

Al-Naimy, H. M. 1978, Ap\&SS, 53, 181

Antokhina E.A., Cherepashchuk A.M., 1985, SvAL, 11, 4A Blundell K.M., Mioduszewski A.J., Muxlow T.W.B., Podsiadlowski P., Rupen M.P., 2001, ApJ, 562, L79

Cherepashchuk A.M., Sunyaev R.A., Fabrika S.N. et al., 2005, accepted by A\&A, (astro-ph/0503352)

Crampton D., Cowley A.P., Hutchings J.B., 1980, ApJ, 235, L131

Crampton D., Hutchings J.B., 1981, ApJ, 251, 604

D’Odorico S., Oosterloo T., Zwitter T., Calvani M., 1991, Nature, 353, 329 
Fabrika S.N., Bychkova L.V., 1990, A\&A, 240, L5

Fabrika S.N., 1997, Ap\&SS, 252, 439

Gies D.R., McSwain M.V., Riddle, R.L., Wang Z., Wiita P.J., Wingert D.W., 2002a, ApJ, 566, 1069

Gies D.R., Huang W., McSwain M.V., 2002b, ApJ, 578, L67

Goranskii V.P., Fabrika S.N., Rakhimov V.Yu., Panferov A.A., Belov A.N., Bychkova L.V., 1997, Astron. Rep., 41, 656

Goranskii V.P., Esipov V.F., Cherepashchuk A.M., 1998, Astron. Rep., 42, 209

Gray, D. F. 1992, The Observation and Analysis of Stellar Photospheres (2nd ed.; Cambridge: Cambridge Univ. Press)

Hillwig T.C., Gies D.R., Huang W., McSwain M.V., Stark M.A., van der Meer A., Kaper L., 2004, ApJ, 615, 422

Horne K., 1986, PASP, 98, 609

Katz J.I., 1981, A\&A, 95, L15

King A.R., Taam R.E., Begelman M.C., 2000, ApJ, 530, L25

Kudritzki R.P., Bresolin F., Przybilla N., 2003, ApJ, 582, L83

Leibowitz E. M., 1984, MNRAS, 210, 279

Lépine S., Moffat A.F.J., St-Louis N. et al, 2000, AJ, 120, 3201

Margon B., 1984, ARA\&A, 22, 507

Newsom G.H., Collins G.W., 1982, ApJ, 262, 714

van den Heuvel E.P.J., Ostriker J.P., Petterson J.A., 1979, A\&A, 81, L7

Zwitter T., Calvani M., D’Odorico S., 1991, A\&A, 251, 92 